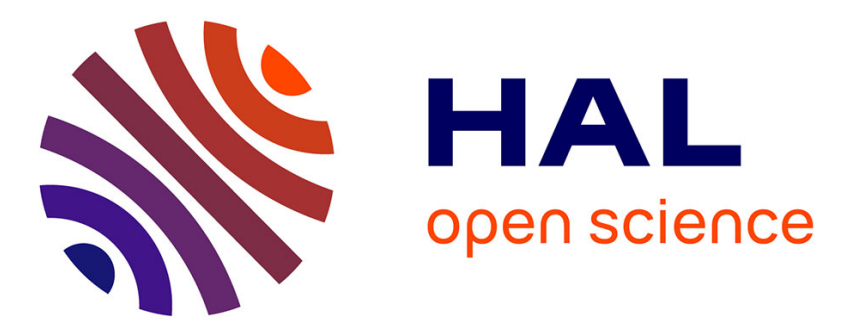

\title{
Stabilization of time-delay systems with a controlled time-varying delay and applications
}

\author{
Wim Michiels, Vincent van Assche, Silviu-Iulian Niculescu
}

\section{To cite this version:}

Wim Michiels, Vincent van Assche, Silviu-Iulian Niculescu. Stabilization of time-delay systems with a controlled time-varying delay and applications. IEEE Transactions on Automatic Control, 2005, 50

(4), pp.493-504. 10.1109/TAC.2005.844723 . hal-02294297

\section{HAL Id: hal-02294297 \\ https://hal.science/hal-02294297}

Submitted on 8 Dec 2019

HAL is a multi-disciplinary open access archive for the deposit and dissemination of scientific research documents, whether they are published or not. The documents may come from teaching and research institutions in France or abroad, or from public or private research centers.
L'archive ouverte pluridisciplinaire HAL, est destinée au dépôt et à la diffusion de documents scientifiques de niveau recherche, publiés ou non, émanant des établissements d'enseignement et de recherche français ou étrangers, des laboratoires publics ou privés. 


\title{
Stabilization of Time-Delay Systems With a Controlled Time-Varying Delay and Applications
}

\author{
Wim Michiels, Member, IEEE, Vincent Van Assche, and Silviu-Iulian Niculescu, Member, IEEE
}

\begin{abstract}
We study the stability of a linear system with a pointwise, time-varying delay. We assume that the delay varies around a nominal value in a deterministic way and investigate the influence of this variation on stability. More precisely we are interested in characterizing situations where the time-varying delay system is stable, whereas the system with constant delay is unstable. Our approach consists of relating the stability properties of a system with a fast varying point-wise delay with these of a time-invariant system with a distributed delay. Then we can use frequency domain methods to analyze the problem and to derive stability criteria. The results are first illustrated with two theoretical examples. Then, we study a model of a variable speed rotating cutting tool. Based on the developed theory, we thereby provide both a theoretical explanation and a quantitative analysis tool for the beneficial effect of a variation of the machine speed on enhancing stability properties, which was reported in the literature.
\end{abstract}

Index Terms-Delay, nonautonomous systems, stability of variable speed machines.

\section{INTRODUCTION}

$\mathbf{T}$ HIS paper concerns the stability analysis of a linear system with a time-varying delay, of the form

$$
\dot{x}(t)=A x(t)+B x(t-\tau(t))
$$

under appropriate initial conditions. We assume that the timedelay varies around a nominal value $\tau_{0}$ in a deterministic way

$$
\tau(t)=\tau_{0}+\delta f(\Omega t)
$$

where $f$ is a periodic function with zero mean and $\delta$ and $\Omega$ are parameters, determining the amplitude and frequency of the variation. We investigate the influence of the variation of the time-delay on the stability of (1).

This problem is inspired by applications in mechanical engineering. More precisely, in the study of the dynamics of rotating

W. Michiels is with the Department of Computer Science, K. U. Leuven, 3001 Heverlee, Belgium (e-mail: Wim.Michiels@cs.kuleuven.ac.be).

V. Van Assche is with the Laboratoire des Technologies Innovantes (LTI), the Université de Picardie-Jules Verne, 02880 Cuffies-Soissons, France (e-mail: Vincent.Vanassche@u-picardie.fr).

S.-I. Niculescu is with HEUDIASYC (UMR-CNRS 6599), Université de Technologie de Compiègne, Centre de Recherches de Royallieu, 60205 Compiègne, France (e-mail: Silviu.Niculescu@ @ds.utc.fr). cutting machines one encounters models of the form (1), where the time-delay represents the time taken by the cutting inserts for one revolution of the workpiece. Therefore, the time-delay is proportional to $1 / N$ with $N$ the rotational speed of the machine. A commonly used way to enlarge the stability region of the steady-state solution of rotational machines and, in this way, preventing undesirable oscillations or chatter, consists of fast modulating the speed around its nominal value [8], [25]. Such a modulation of the speed precisely corresponds to a modulation of the time-delay $\tau$ in (1) of the form (2). Both experimental results and numerical computations have demonstrated the beneficial effect of the variation of the machine speed; see, e.g., [8] and the references therein.

Whereas the effects of constant delays on the systems' dynamics have been largely treated in the literature (see, for instance, [21], [5], and the references therein), the time-varying delay needs a deeper analysis since its presence may induce complex behaviors: see, for example, the so-called quenching phenomenon, as suggested and discussed by Louisell in [12], where he proposed some examples of delay intervals for which the constant and time-varying delays have "opposite" effects: stable for the time-varying delay and unstable for the constant one, and vice versa.

The aim of this paper is to handle the time-varying delay systems mentioned above using an appropriate comparison system combined with some frequency-domain techniques. To the best of the authors' knowledge, there does not exist any similar analysis result in the literature. More explicitly, the stability analysis of (1)-(2) and the comparison with the stability properties of the associated constant delay system

$$
\dot{x}(t)=A x(t)+B x\left(t-\tau_{0}\right)
$$

- to analyze the effect of the delay variation — are performed in two steps.

- $\quad$ First, we show that from a stability point of view, the system (1)-(2) behaves as a time-invariant system with a distributed delay, provided the frequency of variation of the delay, $\Omega$, is large compared to the system's dynamics. Such a condition is satisfied in the applications mentioned previously.

- The second step then consists of comparing the stability of this distributed delay system, whose analysis is more tractable (since it allows, e.g., frequency domain techniques), with the stability of the associated constant delay system. 
While the variation of the delay in (1)-(2) could even be interpreted as a control action, as in the inspiring mechanical application, in the existing literature on time-delay systems a delay variation is always treated as an uncertainty, using an appropriate model transformation of the original system or the definition of some "fictitious" interconnected systems (see, for instance, [21], [5], and the references therein). For a nominally stable system, typically upper/lower bounds on the delay variation and/or its derivative guaranteeing stability are determined. In the time-varying delay case, we mention the Lyapunov based approach [9], some comparison system methodology [23], [4], and the integral quadratic constraint approach [5] to derive such (upper, lower, or derivative) bounds.

A deliberate fast periodic forcing of the delay (2) can be seen as an application of vibrational control. In [11] and the references therein, extensions of the classical averaging theory for time-varying ordinary differential equations toward classes of time-delay systems and the application to vibrational control are considered. In [16], output feedback controllers with oscillatory gains are analyzed. These works do however not treat the case where the time-dependence of the vector-field precisely comes from a variation of the delay.

The structure of the paper is as follows. In Section II, we relate stability properties of (1)-(2) with those of a time-invariant distributed delay system. In Section III, we study the stability of this distributed delay system and compare its properties with the constant delay system (3). In Section IV, we present some illustrations of the main results. After analyzing two theoretical examples, we study a model for a variable speed cutting machine. Section V summarizes the main contributions of the paper. The notations are standard.

\section{Distributed Delays as ApProximation of FAST Time-VARYING POINT-Wise DELAYS}

The main result of the paper relates the stability of a system with a fast varying point-wise delay with the stability of an autonomous system with distributed delay.

Theorem 1: Consider

$$
\begin{aligned}
\dot{x}(t) & =A x(t)+B x(t-\tau(t)) \\
\tau(t) & =\tau_{0}+\delta f(\Omega t)
\end{aligned}
$$

where $x \in \mathbb{R}^{n}, f: \mathbb{R} \rightarrow[-1,1]$ is a periodic function, with zero mean and period $2 \pi, \max f=1$, and $\min f=-1$. Further, let $\delta, \tau_{0}, \Omega \in \mathbb{R}_{0}^{+}$, and $\delta \leq \tau_{0}$. Let the integrable function ${ }^{1}$ $w:[-1,1] \rightarrow \mathbb{R}^{+}$be defined by the relation

$$
\int_{-1}^{1} \alpha(t) w(t) d t=\frac{1}{2 \pi} \int_{0}^{2 \pi} \alpha(f(t)) d t
$$

for every continuous function $\alpha:[-1,1] \rightarrow \mathbb{R}$. If the comparison system

$$
\dot{x}(t)=A x(t)+B \int_{t-\tau_{0}-\delta}^{t-\tau_{0}+\delta} \frac{w\left(\left(\theta-t+\tau_{0}\right) / \delta\right)}{\delta} x(\theta) d \theta
$$

\footnotetext{
${ }^{1}$ In fact, $w$ represents a positive density measure.
}

is asymptotically stable, then (4) is globally uniformly asymptotically stable for large values of the parameter $\Omega$.

For sake of conciseness we postpone a detailed proof to the Appendix. However, because of the nonstandard proof technique, we now outline its main elements.

Sketch of Proof: The existence of an integrable function $w$ satisfying (5) is shown using the change of measure property and the Radon-Nikodym theorem.

The stability assertion follows from an application of the trajectory based proof technique, developed in [18] and [19] for the stability analysis of ordinary differential equations and extended toward classes of delay differential equations in [20]. This proof technique relates closeness results for trajectories (in the sense of uniform convergence of trajectories on compact time-intervals) with stability results (which involve the behavior of trajectories on infinite time-intervals). The main steps are as follows.

1) We prove that trajectories of (4) and (6) with matching initial conditions uniformly converge to each other on compact time-intervals as the parameter $\Omega$ tends to infinity. This is done by estimating the difference of solutions of (4) and (6) at time-instants later than the initial time, and involves the application of a generalization of the celebrated Gronwall Lemma.

2) This closeness result of trajectories of (6) and (4) is linked with stability assertions. By a slight generalization of [18, Th. 1], one can conclude from the exponential stability of (6) and the closeness result that the null solution of system (4) is practically uniformly asymptotically stable (see the Appendix for a precise definition).

3) Practical uniform asymptotic stability of the null solution of (4) implies global uniform asymptotic stability by a scaling property of its solutions.

Remark: From (5), one can interpret $w$ as the probability distribution of $f(\zeta)$, where $\zeta$ is uniformly distributed over the interval $[0,2 \pi]$.

Remark: Under the conditions of the theorem, the stability of (4) is only guaranteed if $\Omega$ is sufficiently large. Explicit bounds on $\Omega$ may be obtained from theoretical considerations (see, e.g., [17]), but these bounds are typically conservative. Therefore, we advise to determine a threshold based on numerical simulation.

The stability of the time-invariant comparison system (6) is determined by the roots of its characteristic equation

$$
\operatorname{det}\left(s I-A-B e^{-s \tau_{0}} g(s \delta)\right)=0
$$

where

$$
g(s)=\int_{-1}^{1} e^{s t} w(t) d t
$$

We now list some general properties of the functions $w$ and $g$, which will be useful in the rest of this paper.

The weight function $w:[-1,1] \rightarrow \mathbb{R}^{+}$always satisfies

$$
\int_{-1}^{1} w(t) d t=1 \quad \int_{-1}^{1} t w(t) d t=0
$$


TABLE I

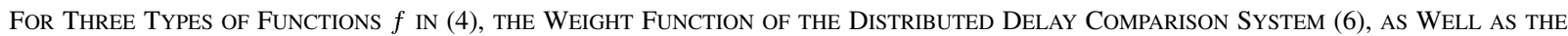
Correction Term $g(s)$ in the Characteristic $(8)$ Are Shown. $J_{0}(\cdot)$ Denotes the Bessel Function of the First Kind of Order ZERO, $h(\cdot)$ IS THE DIRAC IMPULSE FUNCTION

\begin{tabular}{c|l|l}
\hline \hline$f$ & $w$ & $g$ \\
\hline$f_{1}(t)= \begin{cases}\frac{2}{\pi}\left(t-\frac{\pi}{2}\right), & t \in[0, \pi) \\
\frac{2}{\pi}\left(\frac{3 \pi}{2}-t\right), & t \in[\pi, 2 \pi) \\
\text { (sawtooth) }\end{cases}$ & $w_{1}(t)=\frac{1}{2}$ & $g_{1}(s)= \begin{cases}\frac{\sinh (s)}{s}, & s \neq 0 \\
1, & s=0\end{cases}$ \\
\hline$f_{2}(t)=\sin (t)$ & $w_{2}(t)=\frac{1}{\pi \sqrt{1-t^{2}}}$ & $g_{2}(s)=J_{0}(j s)$ \\
\hline$f_{3}(t)= \begin{cases}1, & t \in[0, \pi) \\
-1, & t \in[\pi, 2 \pi) \\
\text { (square wave) }\end{cases}$ & $w_{3}(t)=\frac{(h(t-1)+h(t+1))}{2}$ & $g_{3}(s)=\cosh (s)$ \\
\hline \hline
\end{tabular}

take $\alpha \equiv 1$, respectively $\alpha(t)=t$ in (5). Since $w$ is integrable, the function $g: \mathbb{C} \rightarrow \mathbb{C}$ is smooth, and

$$
\frac{d^{k} g}{d s^{k}}(s)=\int_{-1}^{1} t^{k} e^{s t} w(t) d t, \quad k \geq 0
$$

On the imaginary axis, $g$ has a modulus smaller than one since for all $\omega \in \mathbb{R}$

$$
|g(j \omega)|=\left|\int_{-1}^{1} e^{j \omega t} w(t) d t\right| \leq \int_{-1}^{1}|w(t)| d t=1 .
$$

Furthermore, when $w$ is symmetric around the origin, i.e., $w(-t)=w(t), \forall t \in[-1,1]$, then $g(j \omega)$ is real, following from:

$$
g(-j \omega)=\int_{-1}^{1} e^{-j \omega t} w(t) d t=\int_{-1}^{1} e^{j \omega t} w(-t) d t=g(j \omega)
$$

As an illustration we show for different types of functions $f$ in (4) the corresponding weight function $w$ of the distributed delay of comparison system (6) and the function $g$ in Table I.

\section{STABILITY OF THE COMPARISON SYSTEM}

The next step consists of relating the stability of the comparison system (6) with the stability of the constant delay system

$$
\dot{x}=A x(t)+B x\left(t-\tau_{0}\right)
$$

Notice that (6) can be seen as a modification of (11) by "spreading out" the point-wise delay over an interval of length $2 \delta$. If this leads to larger stability regions in a parameter space, then, by virtue of Theorem 1, a fast forcing of the delay will also lead to larger stability regions.

First, we investigate the stability of (6) for small values of $\delta$. Then, we relate the obtained result with the sensitivity of stability w.r.t. changes of the nominal delay $\tau_{0}$. Finally, we comment on numerical tools for computing the right most eigenvalues of the comparison system (6).

\section{A. Behavior of the Comparison System for Small $\delta$}

We investigate how the eigenvalues of (6) or, equivalently, the roots of

$$
H(s, g(s \delta)):=\operatorname{det}\left(s I-A-B e^{-s \tau_{0}} g(s \delta)\right)=0
$$

behave for small values of $\delta \geq 0$. For $\delta=0$, (12) simplifies to

$$
\operatorname{det}\left(s I-A-B e^{-s \tau_{0}}\right)=0
$$

the characteristic equation of (11).

Because $g$ is a smooth function with $g(0)=1$, roots of (12) are continuous at each value of parameter $\delta \geq 0$. For a given simple root $s_{0}$ of (13), this implies the existence of a root function $r(\delta)$ of (12), satisfying $r(0)=s_{0}$ and

$$
H(r(\delta), g(r(\delta) \delta))=0
$$

To compute the sensitivity of the root $s_{0}$ w.r.t. $\delta$, we differentiate (14), leading to

$$
\begin{aligned}
r^{\prime}(0) & =0 \\
r^{\prime \prime}(0) & =-\frac{\frac{\partial H}{\partial g}\left(s_{0}, 1\right)}{\frac{\partial H}{\partial s}\left(s_{0}, 1\right)} g^{\prime \prime}(0) s_{0}^{2}
\end{aligned}
$$

where $g^{\prime \prime}(0)=\int_{-1}^{1} t^{2} w(t) d t>0$.

The property (15) is due to the zero mean assumption on the delay forcing function $f$, which is expressed in

$$
g^{\prime}(0)=\int_{-1}^{1} t w(t) d t=0
$$

As a consequence, the behavior of the eigenvalues for small $\delta$ is determined by the second-order derivative $r^{\prime \prime}(0)$. A stability related corollary is as follows.

Proposition 1: Assume that the rightmost eigenvalues of (11) are simple and on the imaginary axis. Denote them by $j \omega_{i}, i=1, m$. If

$$
\Re\left\{\frac{\frac{\partial H}{\partial g}\left(j \omega_{i}, 1\right)}{\frac{\partial H}{\partial s}\left(j \omega_{i}, 1\right)} g^{\prime \prime}(0) \omega_{i}^{2}\right\}<0, \quad i=1, m
$$

then (6) is asymptotically stable for small values of $\delta$. 
Proof: Choose $\sigma>0$ such that, except for the eigenvalues on the imaginary axis, (13) has no zeros in $\mathbb{C}_{-\sigma}^{+}:=\{s \in \mathbb{C}$ : $\Re(s) \geq-\sigma\}$. In this half plane, the roots of (12) satisfy

$$
\begin{aligned}
|s| & \leq|A|+|B|\left|e^{-s \tau_{0}} g(s \delta)\right| \\
& \leq|A|+|B| \int_{-1}^{1}\left|e^{\left(\delta t-\tau_{0}\right) s}\right||w(t)| d t \\
& \leq|A|+|B| \int_{-1}^{1}\left|e^{\sigma \tau_{0}}\right||w(t)| d t=|A|+|B| e^{\sigma \tau_{0}} .
\end{aligned}
$$

Therefore, there exists a compact set $S$ containing all the roots of (12) in the half plane $\mathbb{C}_{-\sigma}^{+}$. From the continuity of $g$ and $g(0)=1$, it follows that

$$
\operatorname{det}\left(s I-A-B e^{-s \tau_{0}} g(s \delta)\right) \rightarrow \operatorname{det}\left(s I-A-B e^{-s \tau_{0}}\right)
$$

as $\delta \rightarrow 0$, uniformly on $S$. By [15, Th. A1], a variant of Rouché's theorem, this implies that for small $\delta$ both (entire) functions have the same number of zeros in $S$, and as a consequence in $\mathbb{C}_{-\sigma}^{+}$. Combining this fact with (17), which expresses that the roots lying on the imaginary axis for $\delta=0$ move to the open left half plane when slightly increasing $\delta$, yields that all the roots of (12) lie in the open left-half plane for sufficiently small values of $\delta$.

In the next section, we will study classes of parametrized delay systems, for which condition (17) is always satisfied in case of eigenvalues on the imaginary axis. This means that stability regions in the parameter space become larger when increasing $\delta$ from zero. Indeed, internal points of a stability region in the parameter space correspond to an asymptotically stable system, which remains asymptotically stable when increasing $\delta$ from zero (continuity argument), whereas points on the boundaries of a stability region, if any, correspond to a system with rightmost eigenvalues on the imaginary axis, which becomes asymptotically stable under the conditions of Proposition 1.

Finally, notice that parameter values corresponding to a zero eigenvalue are invariant w.r.t. $\delta$, following from $g(0)=1$.

\section{B. Relation With Delay Sensitivity}

To reveal a relation between the sensitivity of a root $s_{0}$ of (12) for $\delta=0$, i.e., an eigenvalue of (11), w.r.t. changes of $\delta$ and its sensitivity w.r.t. the nominal delay value $\tau_{0}$, extensively studied in [21] and the references therein, one can study the root function

$$
r(\delta, \tau)
$$

satisfying (12) and $r\left(0, \tau_{0}\right)=s_{0}$. A simple computation shows that

$$
\frac{\partial^{2} r}{\partial \delta^{2}}\left(0, \tau_{0}\right)=-s_{0} g^{\prime \prime}(0) \frac{\partial r}{\partial \tau}\left(0, \tau_{0}\right)
$$

Since $g^{\prime \prime}(0)$ is positive, a consequence for the case of roots on the imaginary axis is as follows.

Proposition 2: Assume that $r\left(0, \tau_{0}\right)=j \omega, \omega>0$. Then

$$
\Re\left(\frac{\partial^{2} r}{\partial \delta^{2}}\left(0, \tau_{0}\right)\right)<0 \Leftrightarrow \Im\left(\frac{\partial r}{\partial \tau}\left(0, \tau_{0}\right)\right)<0 .
$$

Thus, the behavior of the real parts of eigenvalues on the imaginary axis when changing $\delta$ is determined by the behavior of their imaginary parts when changing the nominal delay.

This proposition and (18), along with Theorem 1, allow to retrieve stability information for the time-varying delay case directly from known results concerning sensitivity of stability w.r.t. the nominal delay value. This will be illustrated with the second example of the next section.

\section{Computational Tools}

Whereas the aforementioned analytical computations allow to study the stability of the comparison system (6) for small values of $\delta$ and to gain insight in the problem, quantitative stability information for any value of $\delta$ can be obtained by numerically computing the rightmost eigenvalues.

When the weight function $w$ of the distributed delay in (6) is bounded, both the numerical scheme proposed in [1], which relies on a discretization of the infinitesimal generator of the delay equation, as the numerical scheme of [13], which involves a discretization of the time-integration operator, can be used to compute the rightmost eigenvalues. For the specific weight functions $w=w_{3}$, respectively $w=w_{1}$, defined in Table I, tools for delay equations with point-wise delays, such as the software package DDE-BIFTOOL [3] are applicable. In the latter case, the distributed delay with constant weight function in (6) can be removed by a model transformation, yielding an equation with only point-wise delays, as explained in [14]. A nice feature of DDE-BIFTOOL package is its continuation facility which allows to compute stability regions in a two-parameter space semiautomatically.

\section{ILLUSTRATIVE EXAMPLES}

\section{A. First-Order System}

Consider the scalar equation

$$
\dot{x}(t)=a x(t)+b x(t-1-\delta f(\Omega t))
$$

whose stability regions in the $(a, b)$ plane for $\delta=0$ were determined in, e.g., [2]. The distributed delay comparison system is given by

$$
\dot{x}=a x(t)+b \int_{t-1-\delta}^{t-1+\delta} \frac{w((\theta-t+1) / \delta)}{\delta} x(\theta) d \theta
$$

with characteristic equation

$$
H(s, g(s \delta))=s-a-b e^{-s} g(s \delta)=0 .
$$




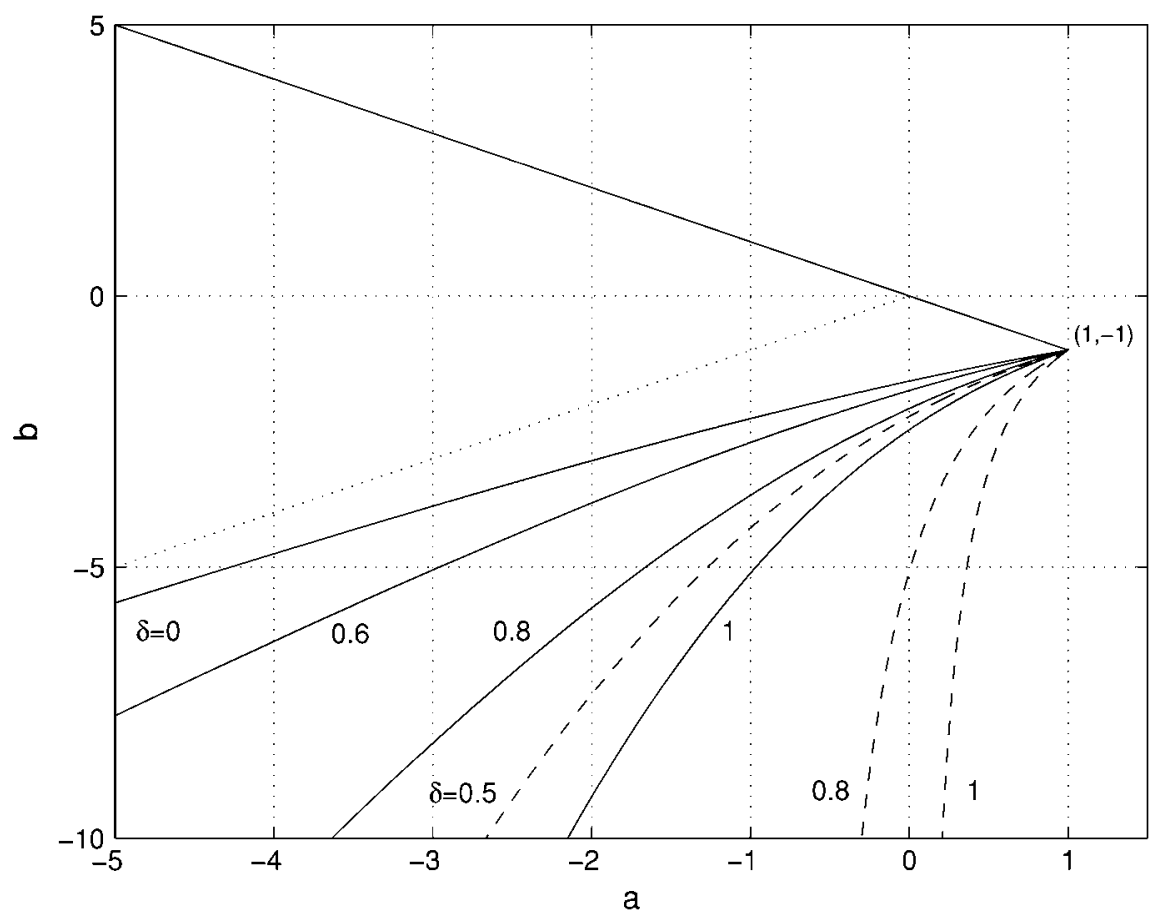

Fig. 1. Stability region of (20) in the $(a, b)$ plane for different values of $\delta$. The solid curves correspond to $g(s)=g_{1}(s)$; the dashed curves to $g(s)=g_{3}(s)$. The analytical expressions are given by (21)-(24).

Applying (16) to an imaginary root $s_{0}=j \omega$ of $H(s, g(0))$ and taking the real part give

$$
\begin{aligned}
\Re\left(r^{\prime \prime}(0)^{-1}\right) & =\Re\left[\frac{\frac{\partial H}{\partial g}(j \omega, 1)}{\frac{\partial H}{\partial s}(j \omega, 1)} g^{\prime \prime}(0) \omega^{2}\right]^{-1} \\
& =\frac{1}{g^{\prime \prime}(0) \omega^{2}}\left(-1+\frac{a}{a^{2}+\omega^{2}}\right) \\
& =\frac{1}{g^{\prime \prime}(0) \omega^{2}}\left(-1+\frac{a}{b^{2}}\right) .
\end{aligned}
$$

This quantity is negative for purely imaginary eigenvalues corresponding to values of $a$ and $b$ on the stability boundary, since such parameter values satisfy $a / b^{2}<1$, see [2]. Therefore, the stability region of $(20)$ in the $(a, b)$ plane enlarges when $\delta$ is increased from zero. Under the assumption of a symmetric weighting function, i.e., $w(t)=-w(t)$, this stability region can easily be computed analytically using the method of $D$-subdivision [10], to yield

$$
a<1, \quad \bar{b}(a)<b<-a
$$

where the relation $\bar{b}(a)$ is implicitly defined by the curve

$$
\omega \in(0, \pi) \rightarrow(a(\omega), \bar{b}(\omega))
$$

with (23) and (24), as shown at the bottom of the page. Notice that for any $\omega \leq \bar{\omega}, g(j \omega)$ is a positive real number, smaller than one, following from (10), (9), and $g(0)=1$. Furthermore, the specific choices of $g$, defined in Table I, satisfy

$$
g_{1}(j \omega)>g_{2}(j \omega)>g_{3}(j \omega) \quad \forall \omega \in[0, \pi] .
$$

From (23) and (25), one can conclude that the largest stability region is obtained for the case $\left(f_{3}, w_{3}, g_{3}\right)$, where all the weight of the distributed delay lies in end-points of the interval.

In Fig. 1, we show the stability region of $(20)$ in the $(a, b)$ plane for different values of $\delta$ and $g(s)=g_{1}(s)$, respectively $g(s)=g_{3}(s)$. In the latter case and for $\delta>1 / 2$, we have $\bar{b}(a)=-\infty$ for $a \leq(\pi) /(2 \delta \tan (\pi / 2 \delta))$. In Fig. 2(a), we plot the real parts of the rightmost eigenvalues of $(20)$ with $(a, b)=$ $(-1,-4)$ as a function of $\delta$. The solid curves correspond to $g=g_{1}(s)$, the dashed curves to $g=g_{3}(s)$. In Fig. 2(b), we

and

$$
\begin{aligned}
& a(\omega)= \begin{cases}\frac{\omega}{\tan \omega}, & \omega \neq \pi / 2 \\
0, & \omega=\pi / 2\end{cases} \\
& \bar{b}(\omega)= \begin{cases}-\frac{\omega}{\sin \omega} \frac{1}{g(j \omega \delta)}, & \omega \delta<\bar{\omega} \\
-\infty, & \text { otherwise }\end{cases}
\end{aligned}
$$

$$
\bar{\omega}=\left\{\begin{array}{ll}
+\infty, & g(j \omega) \neq 0 \\
\min \{\omega \in[0, \pi]: g(j \omega)=0\}, & \text { otherwise }
\end{array} \quad \forall \omega \in[0, \pi]\right.
$$




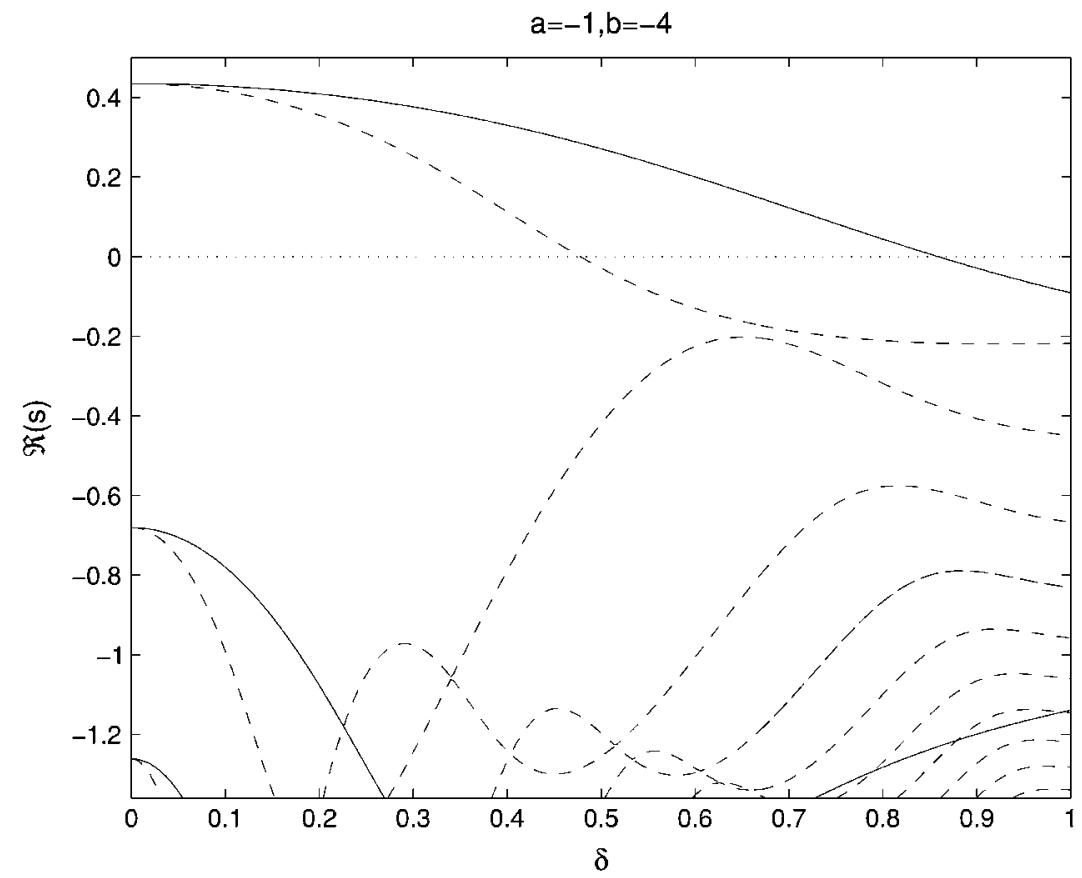

(a)
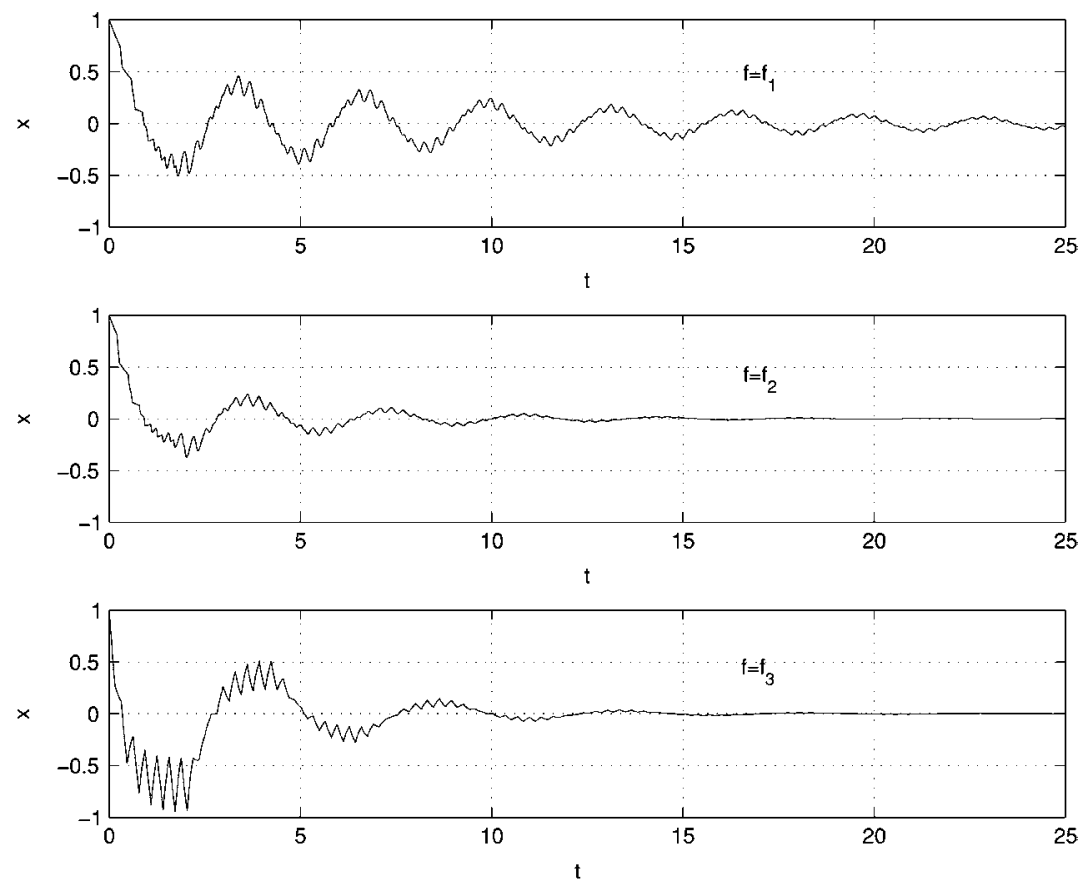

(b)

Fig. 2. (a) Real parts of the rightmost eigenvalues of (20) with $(a, b)=(-1,-4)$ as a function of $\delta$. The solid curves correspond to $g=g_{1}(s)$, the dashed curves to $g=g_{3}(s)$. (b) Simulated trajectories of the original system (19) with $(a, b)=(-1,-4), \delta=1, \Omega=20, x_{0} \equiv 1$, and $f=f_{1}, f_{2}, f_{3}$ (above, middle, below).

show simulated trajectories of the corresponding system with time-varying delay (19).

\section{B. Delayed Feedback Stabilized Oscillators}

Consider a single-input-single-output system with rational transfer function

$$
\frac{Y(s)}{U(s)}=\frac{P(s)}{Q(s)}
$$

where $\operatorname{deg} P(s)<\operatorname{deg} Q(s)$, in closed-loop with a delayed feedback control law

$$
u(t)=-k y\left(t-\tau_{0}-\delta f(\Omega t)\right) .
$$

For $\delta=0$, this stabilization problem was studied in [22] and a procedure to determine a stabilizing pair $\left(k, \tau_{0}\right)$ (if any) was presented. 


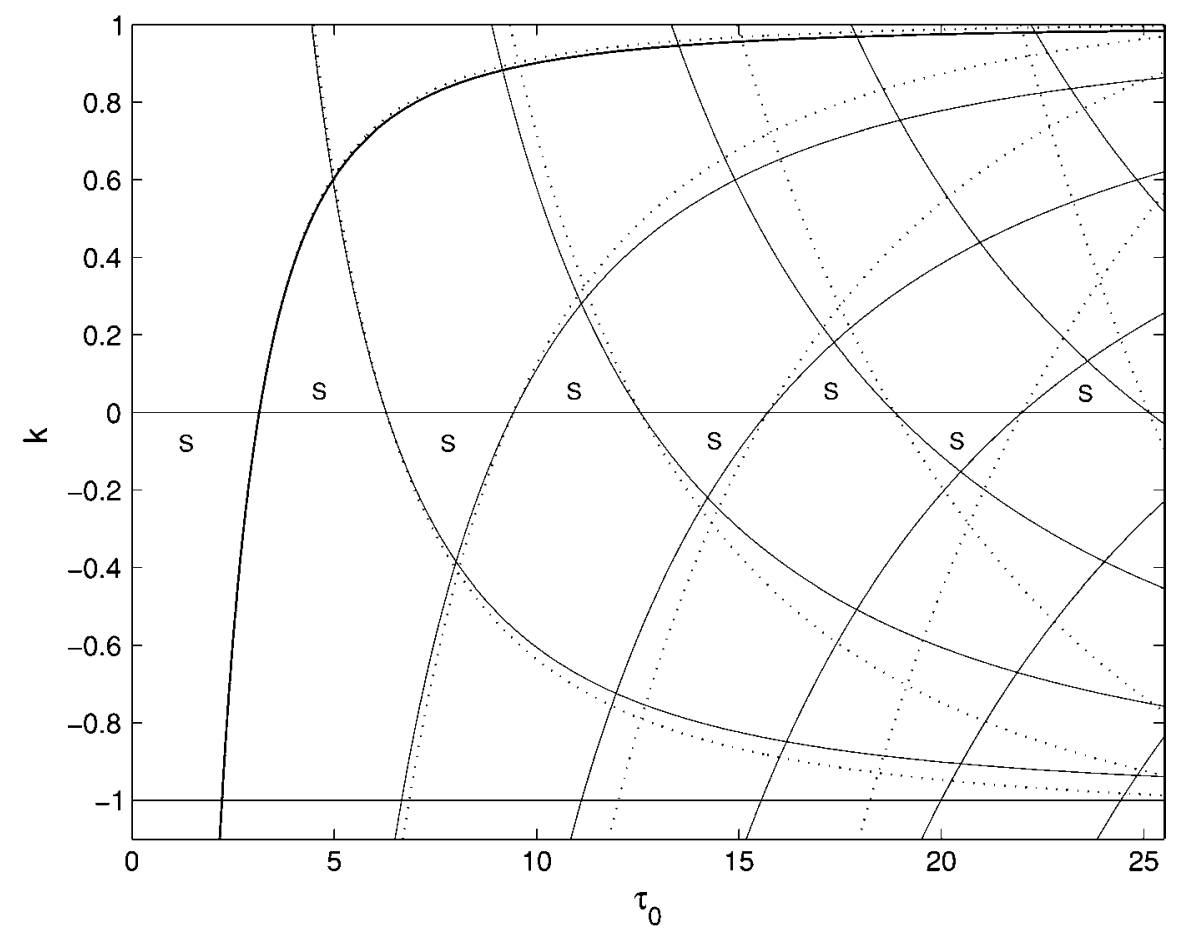

Fig. 3. Solid curves separate stability-instability regions of (29) in the $(k, \tau)$-plane for $\delta_{\text {rel }}=0$. Stable regions are indicated with "S." For $\delta_{\text {rel }}=0.05$ the stability regions of (29) are separated with the dotted curves.

The characteristic equation of the distributed delay comparison system of (26)-(27) is given by

$$
H(s, g(s \delta)):=Q(s)+k P(s) e^{-s \tau_{0}} g(s \delta)=0 .
$$

When applying (16) to an imaginary root $s_{0}=j \omega$ of $H(s, g(0))$, some simple computations yield

$$
\begin{aligned}
& \Re\left(r^{\prime \prime}(0)^{-1}\right)=-\frac{1}{\omega^{2} g^{\prime \prime}(0)} \\
& \quad \times\left(\tau+\Re \frac{Q^{\prime}(j \omega) Q(-j \omega)-k^{2} P^{\prime}(j \omega) P(-j \omega)}{|Q(j \omega)|^{2}}\right) .
\end{aligned}
$$

For more details, see the proof of [22, Th. 5], where one computes the sensitivity of eigenvalues to changes of the nominal delay value $\tau_{0}$, and apply property (18).

When $P(s)$ is constant and $Q(s)$ even, expression (28) simplifies to

$$
\Re\left(r^{\prime \prime}(0)^{-1}\right)=-\frac{\tau}{\omega^{2} g^{\prime \prime}(0)}<0
$$

thus increasing $\delta$ from zero leads to larger stability regions. As an illustration, we show in Fig. 3 stability regions of

$$
s^{2}+1+k e^{-s \tau_{0}} g_{3}\left(s \delta_{\text {rel }} \tau_{0}\right)=0
$$

in the $\left(\tau_{0}, k\right)$ space for two different values of $\delta_{\text {rel }}:=\delta / \tau_{0}$. Notice that (29) concerns the case of an output feedback controlled second-order oscillator, $P(s) / Q(s)=1 /\left(s^{2}+1\right)$. The nominal delay $\tau_{0}$ is considered also as a system parameter and $\delta_{\text {rel }}$ concerns the relative width of the interval over which the delay is varied.

\section{Variable Spindle Speed Cutting Machines}

The following equation, taken from [8]:

$$
\begin{array}{r}
\ddot{x}(t)+2 \xi \omega_{n} \dot{x}(t)+\omega_{n}^{2} x(t)=\frac{k}{m}(x(t-\tau(t))-x(t)) \\
x \in \mathbb{R}
\end{array}
$$

models one mode of a mechanical rotational cutting process, where $x$ represents the deflection of the machine tool and/or workpiece, $\omega_{n}$ the natural frequency, $\xi$ the damping ratio and $m$ the modal mass. The term $k(x(t-\tau(t))-x(t))$, with $k>0$ the cutting force coefficient, models the cutting force, which depends on the time $\tau$, taken by the cutting insert for one revolution of the workpiece. Clearly the time-delay is proportional to $1 / N$ with $N$ the rotational speed of the machine. In [8] one assumes that this speed is varied around a nominal value in the following way:

$$
N(t)=N_{0}+N_{1} \sin (\Omega t), \quad N_{0}, N_{1} \in \mathbb{R}
$$

which precisely corresponds to a modulation of the time-delay of the form

$$
\tau(t)=\tau_{0}+\delta f(\Omega t) .
$$

Based on the theory developed in this paper and the model (30), we now give an explanation for the beneficial effect of a high frequency modulating of the machine speed on increasing stability regions.

The characteristic equation of the distributed delay comparison system of (30)-(31) is given by

$$
H(s, g(s)):=s^{2}+2 \xi \omega_{n} s+\omega_{n}^{2}+\frac{k}{m}\left(1-e^{-s \tau} g(s \delta)\right)=0 .
$$




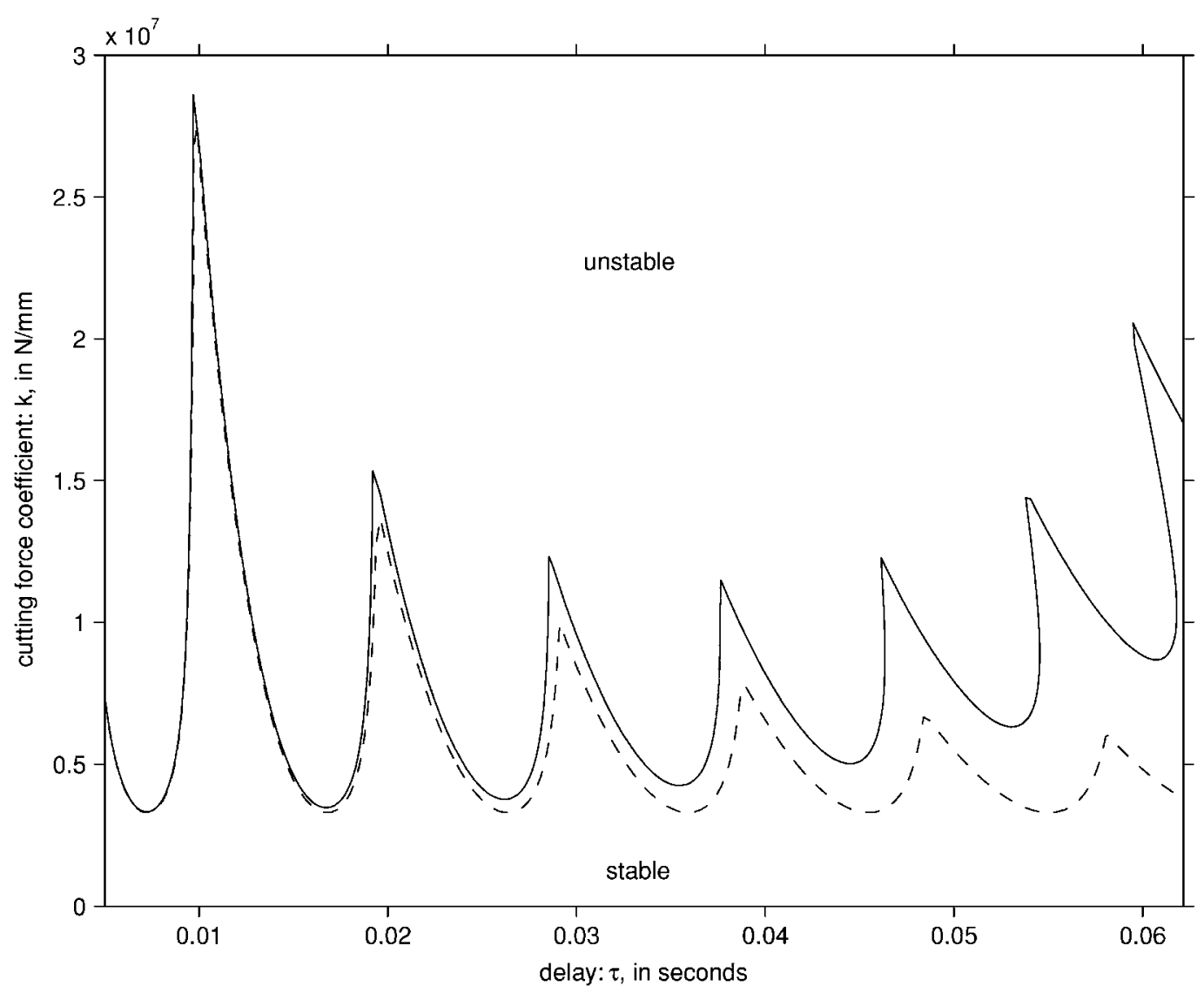

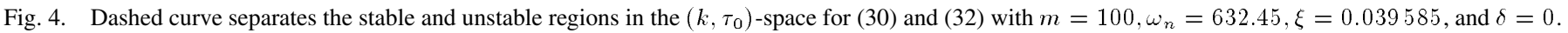
The solid curve separates the stable and unstable regions for the distributed delay comparison system with $\delta=0.05 \tau_{0}$.

Applying the sensitivity formula (16) to an imaginary root $j \omega$ yields

$\Re\left(r^{\prime \prime}(0)^{-1}\right)$
$=-\frac{1}{g^{\prime \prime}(0) \omega^{2}}\left(\tau+2 \xi \omega_{n}^{2} \frac{\omega^{2}+\left(\omega_{n}-k / m\right)^{2}}{\left(\omega^{2}-\left(\omega_{n}^{2}+k / m\right)\right)^{2}+4 \xi^{2} \omega_{n}^{2} \omega^{2}}\right)$

which is strictly negative for any value of the system parameters and any delay forcing function $f$. Therefore, the stability region of the steady state solution can always be enlarged by "distributing" the point-wise delay over an interval, or, by virtue of Theorem 1, by modulating the point-wise delay (speed).

To illustrate this, we compute the stability limits for the system (30) and for the comparison system corresponding to a modulation of the delay

$$
\tau(t)=\tau_{0}+\delta f_{1}(\Omega t)
$$

where $f_{1}(t)$ is the sawtooth function described in Table I. With this choice, the kernel of the distributed delay of the comparison system is a constant. Then, as explained in [14], we can use the DDE-BIFTOOL package [3] to compute accurately the stability limits which appear in Fig. 4 . Note that the $k$ coefficient depends on the width of the cutting tool and on the nominal depth of cut. Therefore, the variation of the rotating speed allows to use a larger tool and/or to remove more material at once, especially at lower speed (i.e., larger delay).

\section{CONCLUding REMARKS}

We investigated the effect of a delay variation on the stability of linear time-delay systems. Theorem 1 related the stability of a time-varying point-wise delay system with the stability of a time-invariant distributed delay system. Its proof consisted of an application of the recently developed trajectory-based proof technique, which is time-domain based and relies on a comparison of trajectories. As a mathematical tool, an extension of the Gronwall Lemma to distributed delay systems played an important role.

The stability of the distributed delay comparison system was analyzed by studying the roots of its characteristic equation. For sake of generality, the derived stability criteria, as Proposition 1, were formulated using the correction function $g$ and are thus not restricted to a particular class of delay variations (function $f$ ). The same holds for the stabilizability assertions in the examples. Only for the graphical illustrations particular choices of Table I were used.

We provided three examples where a delay variation always leads to larger stability regions in a parameter space. For the rotating cutting machine application, our results confirmed that a variation of the rotational speed can be used to allow a larger tool and/or to remove more material per turn. Furthermore, we believe that studying the eigenvalues of the distributed delay comparison systems and their numerical computation are useful new quantitative and qualitative tools for analysis and parameter tuning in such problems. 


\section{APPENDIX \\ PROOF OF THE MAIN THEOREM}

We give a detailed Proof of Theorem 1 . This requires a generalization of the Gronwall Lemma, which we consider first.

\section{A. Generalization of the Gronwall Lemma}

Lemma 1: For real numbers $\tau_{2}>\tau_{1}>0$, define $w: \mathbb{R} \rightarrow$ $\mathbb{R}^{+}$as an integrable function satisfying $\int_{-\tau_{0}}^{-\tau_{1}} w(s) d s=1$. If a continuous function $x: \mathbb{R} \rightarrow \mathbb{R}$ satisfies $x(t)=0, \forall t \leq 0$, and

$|x(t)| \leq c_{1}+c_{2} \int_{0}^{t}\left(|x(s)|+\int_{s-\tau_{2}}^{s-\tau_{1}} w(\theta-s)|x(\theta)| d \theta\right) d s$

$\forall t \geq 0$

then it satisfies $|x(t)| \leq c_{1} \exp \left(2 c_{2} t\right), \forall t \geq 0$.

Proof: Define the function $z: \mathbb{R} \rightarrow \mathbb{R}$ by $z(t)=$ $\max _{s \in[t-\Delta \tau, t]}|x(s)|$, where $\Delta \tau=\tau_{2}-\tau_{1}$. The right-hand size of (33) is a nondecreasing function of $t$. Therefore, it is not only an upper bound for $|x(t)|$, but also of $z(t)$ ! Thus, we have for all $t \geq 0$

$$
\begin{aligned}
z(t) & \leq c_{1}+c_{2} \int_{0}^{t}\left(|x(s)|+\int_{s-\tau_{2}}^{s-\tau_{1}} w(\theta-s)|x(\theta)| d \theta\right) d s \\
& \leq c_{1}+c_{2} \int_{0}^{t}\left(z(s)+z\left(s-\tau_{1}\right)\right) d s \\
& \leq c_{1}+2 c_{2} \int_{0}^{t} z(s) d s .
\end{aligned}
$$

By the classical Gronwall Lemma [6, Lemma 3.1, pp. 15-16], one concludes from (34) that

$$
z(t) \leq c_{1} \exp \left(2 c_{2} t\right) \quad \forall t \geq 0
$$

and from the definition of $z$ the assertion of the Lemma follows.

\section{B. Proof of Theorem 1}

First, we show that there always exists an integrable function $w$ satisfying (5). Intuitively, for fixed $f$, the right-hand side of (5) can be considered as the image of the function $\alpha$ under a continuous linear functional over the space $C([-1,1], \mathbb{R})$. Next, following from the Riesz Representation Theorem this image can always be expressed as a weighted integral of the function over the space (left-hand side).

In a different way, since $f$ defined as in the Theorem's statement is measurable (a bounded function on some compact), then for any $\sigma$-finite measure $\mu$ for which the right-hand integral in (5) is properly defined in the $\mathcal{L}^{1}$ sense (see, for instance, [24]), the quantity $f(\mu)$ will define a "new" measure, and we will have the equality (change of measure)

$$
\int_{[0,2 \pi]} \alpha(f(\mu)) d \mu=\int_{[-1,1]} \alpha d(f(\mu))
$$

with respect to the measure $\mu$ for any continuous function $\alpha$, since $f([0,2 \pi]) \subset[-1,1]$, and there exists $\underline{x}, \bar{x} \in[0,2 \pi]$ such that $f(\underline{x})=-1$, and $f(\bar{x})=1$. Here, $f(\mu)$ is defined as follows: for any set $\mathcal{A} \subset[-1,1], f(\mu)(\mathcal{A})=\mu\left[f^{-1}(\mathcal{A})\right]$ (the measure of the inverse image $(\bmod (2 \pi))$; see, for instance, [7]). Notice that the result is still valid if $\alpha$ is integrable, and not necessarily continuous. Next, since $f$ has zero mean, and using the previous definition, we may restrict the class of measures $\mu$ to those measures for which $f(\mu)$ is positive.

Finally, for any measure $\nu, \sigma$-finite which is absolutely continuous with respect to $f(\mu)$ (see [24] for the definitions), there exists a (positive) measure $g$ (integrable function) such that the following condition holds:

$$
\frac{1}{2 \pi} \int_{[-1,1]} \alpha d(f(\mu))=\int_{[-1,1]} \alpha d(g \nu) .
$$

The existence of a such a measure $g$ is guaranteed by the Radon-Nikodym theorem (see [24]). The last term can be rewritten as follows (density measure properties):

$$
\int_{[-1,1]} \alpha d(g \nu)=\int_{[-1,1]} \alpha g d \nu
$$

which simply says that the density $g$ will define the integrable $w$ in (5)

We now come to the stability assertion, where we use the trajectory-based proof technique, developed in [18]-[20].

For a given $t_{0} \in \mathbb{R}$ and $\phi \in \mathcal{C}\left(\left[-\tau_{0}-\delta, 0\right], \mathbb{R}^{n}\right)$, where $\mathcal{C}\left(\left[-\tau_{0}-\delta, 0\right], \mathbb{R}^{n}\right)$ is the Banach space of continuous functions mapping the interval $\left[-\tau_{0}-\delta, 0\right]$ into $\mathbb{R}^{n}$ and equipped with the supremum norm $|\cdot|_{s}$, denote by $t_{m}=\min _{t>t_{0}} t-\tau_{0}-\delta f(\Omega t)$ and define $x_{\Omega}\left(\phi, t_{0}\right)(t)$ as the unique forward solution of (4) with initial condition $x_{\Omega}\left(\phi, t_{0}\right)\left(t_{0}+\theta\right)=\phi(\theta), \forall \theta \in\left[t_{m}-\right.$ $\left.t_{0}, 0\right]$. Analogously, define $z\left(\phi, t_{0}\right)(t)$ as the unique forward solution of (6) with initial condition $z\left(\phi, t_{0}\right)\left(t_{0}+\theta\right)=\phi(\theta), \forall \theta \in$ $\left[-\tau_{0}-\delta, 0\right]$.

For technical reasons, we temporarily restrict the state-space to $\mathcal{C}_{r}\left(\left[-\tau_{0}-\delta, 0\right], \mathbb{R}^{n}\right)$, defined as

$$
\begin{aligned}
& \mathcal{C}_{r}\left(\left[-\tau_{0}-\delta, 0\right], \mathbb{R}^{n}\right) \\
& \quad:=\left\{\phi \in \mathcal{C}\left(\left[-\tau_{0}-\delta, 0\right], \mathbb{R}^{n}\right):|\phi(t)-\phi(s)|\right. \\
& \left.\quad \leq(|A|+|B|)|\phi|_{s}|t-s|, \forall t, s \in\left[-\tau_{0}-\delta, 0\right]\right\} .
\end{aligned}
$$

This is possible because the following invariance property holds along the solutions of (4) and (6):

$$
\begin{array}{r}
\theta \in\left[-\tau_{0}-\delta, 0\right] \rightarrow x_{\Omega}\left(\phi, t_{0}\right)(t+\theta) \in \mathcal{C}_{r}\left(\left[-\tau_{0}-\delta, 0\right], \mathbb{R}^{n}\right) \\
\forall t \geq t_{0} \\
\theta \in\left[-\tau_{0}-\delta, 0\right] \rightarrow z\left(\phi, t_{0}\right)(t+\theta) \in \mathcal{C}_{r}\left(\left[-\tau_{0}-\delta, 0\right],\right. \\
\left.\mathbb{R}^{n}\right) \\
\forall t \geq t_{0}
\end{array}
$$$$
\forall t_{0} \in \mathbb{R} \quad \forall \Omega>0 \quad \forall \phi \in \mathcal{C}_{r}\left(\left[-\tau_{0}-\delta, 0\right], \mathbb{R}^{n}\right)
$$

following from the fact that for all $t \geq t_{0}$

$$
\begin{aligned}
\left|\dot{x}_{\Omega}\left(\phi, t_{0}\right)(t)\right| \leq & |A|\left|x_{\Omega}\left(\phi, t_{0}\right)(t)\right| \\
& +|B|\left|x_{\Omega}\left(\phi, t_{0}\right)\left(t-\tau_{0}-\delta f(\Omega t)\right)\right|
\end{aligned}
$$

and

$$
\begin{aligned}
\left|\dot{z}(t)\left(\phi, t_{0}\right)(t)\right| \leq & |A|\left|z\left(\phi, t_{0}\right)(t)\right| \\
& |B| \max _{\theta \in\left[t-\tau_{0}-\delta, t-\tau_{0}+\delta\right]}\left|z\left(\phi, t_{0}\right)(\theta)\right|
\end{aligned}
$$

where we used $\int_{-1}^{1} w(t) d t=1$. 
The crucial step of the trajectory based approach consists of proving uniform convergence of solutions of (4) and (6) on compact time-intervals, in the following sense:

$$
\begin{aligned}
& \forall T>\quad c>0 \quad \forall \epsilon>0 \exists \bar{\Omega}>0 \text { s.t. } \\
& \left|x_{\Omega}\left(\phi, t_{0}\right)(t)-z\left(\phi, t_{0}\right)(t)\right| \leq \epsilon \quad \forall t \in\left[t_{0}, t_{0}+T\right] \\
& \forall \Omega \geq \bar{\Omega} \quad \forall t_{0} \in \mathbb{R} \quad \forall \phi \in \mathcal{C}_{r}\left(\left[-\tau_{0}-\delta, 0\right], \mathbb{R}^{n}\right) \\
& \text { with }|\phi|_{s} \leq c .
\end{aligned}
$$

Therefore, fix $c, T, \epsilon$. When omitting the explicit dependence of $x_{\Omega}(t)$ and $z(t)$ on the initial condition $\left(\phi, t_{0}\right)$ for notational convenience, we have

$$
x_{\Omega}(t)=\phi(0)+\int_{t_{0}}^{t}\left(A x_{\Omega}(s)+B x_{\Omega}(s-\tau(s))\right) d s
$$

and

$$
\begin{aligned}
z(t)= & \phi(0)+\int_{t_{0}}^{t}(A z(s) \\
& \left.+B \int_{s-\tau_{0}-\delta}^{s-\tau_{0}+\delta} \frac{w\left(\left(\theta-s+\tau_{0}\right) / \delta\right)}{\delta} z(\theta) d \theta\right) d s .
\end{aligned}
$$

The difference satisfies the estimate

$$
\begin{aligned}
& \left|z(t)-x_{\Omega}(t)\right| \leq \int_{t_{0}}^{t}\left(|A|\left|z(s)-x_{\Omega}(s)\right| d s+|B|\right. \\
& \quad \times \int_{s-\tau_{0}-\delta}^{s-\tau_{0}+\delta} \frac{w\left(\left(\theta-s+\tau_{0}\right) / \delta\right)}{\delta} \\
& \left.\quad \times\left|z(\theta)-x_{\Omega}(\theta)\right| d \theta\right) d s+|B||J(t)|
\end{aligned}
$$

where

$$
\begin{array}{r}
J(t)=\int_{t_{0}}^{t}\left(\int_{s-\tau_{0}-\delta}^{s-\tau_{0}+\delta} \frac{w\left(\left(\theta-s+\tau_{0}\right) / \delta\right)}{\delta} x_{\Omega}(\theta) d \theta\right. \\
\left.-x_{\Omega}\left(s-\left(\tau_{0}+\delta f(\Omega s)\right)\right)\right) d s
\end{array}
$$

and using Lemma 1, relation (39) implies

$$
\begin{aligned}
\mid z(t)- & x_{\Omega}(t)|\leq| B|| J(t) \mid \exp (2(|A|+|B|) t) \\
& \leq|B| J_{m} \exp (2(|A|+|B|) T) \quad \forall t \in\left[t_{0}, t_{0}+T\right]
\end{aligned}
$$

where

$$
J_{m}=\max _{\theta \in\left[t_{0}, t_{0}+T\right]}|J(\theta)|
$$

We now derive an upper bound on $J_{m}$. For that, define a sequence of times

$$
t_{i}=t_{0}+2 \pi / \Omega, \quad i \in \mathbb{N}
$$

and choose $k \in \mathbb{N}$ such that $t_{k}<t_{0}+T \leq t_{k+1}$. Some simple computations yield (43), as shown at the bottom of the page.

Because of (36)-(37), there exists a constant $M<0$ such that

$$
\begin{array}{cc}
\left|x_{\Omega}\left(\phi, t_{0}\right)(t)\right|<M \quad\left|z\left(\phi, t_{0}\right)(t)\right|<M \quad \forall t \in\left[t_{0}, t_{0}+T\right] \\
\forall t_{0} \in \mathbb{R} \quad \forall \phi \in C_{r}\left(\left[-\tau_{0}-\delta, 0\right], \mathbb{R}^{n}\right) \quad \text { with }|\phi|_{s}<c .
\end{array}
$$

We now let $\Omega \rightarrow \infty$ or, equivalently, $k \rightarrow \infty$, and reconsider the estimate (43). Following from the definition (5), we have

$$
J_{1}=0
$$

and from (44) and (42)

$$
J_{4} \rightarrow 0 \text { as } k \rightarrow \infty
$$

uniformly w.r.t. $t_{0} \in \mathbb{R}$ and $\phi \in \mathcal{C}\left(\left[-\tau_{0}-\delta, 0\right], \mathbb{R}^{n}\right)$ with $|\phi|_{s}<$ $c$. The estimates (36)-(37) and the restriction of the state space to (35) imply that the functions $t \rightarrow z(t)$ and $t \rightarrow x_{\Omega}(t)$ are Lipschitz on $\left[t_{0}-\tau_{0}-\delta, t_{0}+T\right]$ with Lipschitz constants, which can be chosen independent of $\Omega>0, t_{0} \in \mathbb{R}$, and $\phi \in$

$$
\begin{aligned}
J_{m} \leq & \underbrace{\sum_{i=0}^{k-1}\left|\int_{t_{i}}^{t_{i+1}}\left(\int_{t_{i}-\tau_{0}-\delta}^{t_{i}-\tau_{0}+\delta} \frac{w\left(\left(\theta-s+\tau_{0}\right) / \delta\right)}{\delta} x_{\Omega}(\theta) d \theta-x_{\Omega}\left(t_{i}-\left(\tau_{0}+\delta f(\Omega s)\right)\right)\right) d s\right|}_{J_{1}}|\underbrace{}_{J_{2}} x_{\Omega}(\theta) d \theta-\int_{s-\tau_{0}-\delta}^{s-\tau_{0}+\delta} \frac{w\left(\left(\theta-s+\tau_{0}\right) / \delta\right)}{\delta} x_{\Omega}(\theta) d \theta| d s \\
& +\underbrace{\sum_{i=0}^{k-1} \int_{t_{i}}^{t_{i+1}} \mid \int_{t_{i}-\tau_{0}-\delta}^{t_{i}-\tau_{0}+\delta} \frac{w\left(\left(\theta-s+\tau_{0}\right) / \delta\right)}{\delta}}_{J_{3}} \\
& +\underbrace{\sum_{i=0}^{k-1} \int_{t_{i}}^{t_{i}+1}\left|x_{\Omega}\left(t_{i}-\left(\tau_{0}+\delta f(\Omega s)\right)\right)-x_{\Omega}\left(s-\left(\tau_{0}+\delta f(\Omega s)\right)\right)\right| d s}_{J 4} \\
& +\underbrace{d s}_{\int_{t_{k}}^{t}\left|\int_{s-\tau_{0}-\delta}^{s-\tau_{0}+\delta} \frac{w\left(\left(\theta-s+\tau_{0}\right) / \delta\right)}{\delta} x_{\Omega}(\theta) d \theta-x_{\Omega}\left(s-\left(\tau_{0}+\delta f(\Omega s)\right)\right)\right| d s}
\end{aligned}
$$


$\mathcal{C}\left(\left[-\tau_{0}-\delta, 0\right], \mathbb{R}^{n}\right)$ with $|\phi|_{s}<c$. As a consequence there also exists a constant $L$ such that

$$
J_{2} \leq \sum_{i=0}^{k-1} \int_{t_{i}}^{t_{i+1}} L \cdot\left(s-t_{i}\right) d s=\frac{L}{2} \sum_{i=0}^{k-1}\left(t_{i+1}-t_{i}\right)^{2} .
$$

Since $k \sim \Omega=2 \pi /\left(t_{i+1}-t_{i}\right)$, we have $J_{2} \rightarrow 0$ as $\Omega \rightarrow \infty$, uniformly w.r.t. $t_{0} \in \mathbb{R}$ and $\phi \in \mathcal{C}\left(\left[-\tau_{0}-\delta, 0\right], \mathbb{R}^{n}\right)$ with $|\phi|_{s}<$ $c$. Similarly, one can show that $J_{3} \rightarrow 0$ as $\Omega \rightarrow \infty$, uniformly w.r.t. $t_{0} \in \mathbb{R}$ and $\phi \in \mathcal{C}\left(\left[-\tau_{0}-\delta, 0\right], \mathbb{R}^{n}\right)$ with $|\phi|_{s}<c$.

Putting the previous elements together, we have proven that

$$
J_{m} \rightarrow 0 \text { as } \Omega \rightarrow \infty
$$

uniformly w.r.t. $t_{0} \in \mathbb{R}$ and $\phi \in \mathcal{C}\left(\left[-\tau_{0}-\delta, 0\right], \mathbb{R}^{n}\right)$ with $|\phi|_{s}<c$. Combining (41) and (45) results in (38).

The next step of the trajectory based approach consists of linking the closeness result (38) with stability assertions. By a slight modification ${ }^{2}$ of [18, Th. 1], the exponential stability of (6) and the property (38) imply that the origin of system (4) is practically uniformly asymptotically stable, in the following sense [18, Def. 2].

1) (Semiglobal uniform boundedness):

$$
\begin{aligned}
& \forall c_{1}>0, \exists c_{2}>0, \bar{\Omega}>0 \text { s.t. } \\
& \forall \Omega \geq \bar{\Omega} \quad \forall t_{0} \in \mathbb{R} \forall \phi \in \mathcal{C}_{r}\left(\left[-\tau_{0}-\delta, 0\right], \mathbb{R}^{n}\right), \quad \forall t \geq t_{0} \\
& \text { with }|\phi|_{s} \leq c_{1} .
\end{aligned}
$$

2) (Semiglobal uniform attractivity):

$$
\begin{gathered}
\forall c_{1}>0, c_{2}>0, \exists T>0, \bar{\Omega}>0, \text { s.t. } \\
\qquad\left|x_{\Omega}\left(\phi, t_{0}\right)(t)\right| \leq c_{2} \quad \forall t \geq t_{0}+T \\
\forall \Omega \geq \bar{\Omega} \quad \forall t_{0} \in \mathbb{R} \forall \phi \in \mathcal{C}_{r}\left(\left[-\tau_{0}-\delta, 0\right], \mathbb{R}^{n}\right), \\
\text { with }|\phi|_{s} \leq c_{1} . \quad \text { (47) }
\end{gathered}
$$

3) (Uniform stability):

$$
\begin{array}{r}
\forall c_{2}>0, \exists c_{1}>0, \bar{\Omega}>0 \text { s.t. }\left|x_{\Omega}\left(\phi, t_{0}\right)(t)\right| \leq c_{2} \forall t \geq t_{0} \\
\forall \Omega \geq \bar{\Omega} \forall t_{0} \in \mathbb{R} \forall \phi \in \mathcal{C}_{r}\left(\left[-\tau_{0}-\delta, 0\right], \mathbb{R}^{n}\right), \\
\text { with }|\phi|_{s} \leq c_{1} .
\end{array}
$$

This result can be strengthened. From (47) and (46), one can conclude that (with initial conditions restricted to $\mathcal{C}_{r}\left(\left[-\tau_{0}-\right.\right.$ $\left.\delta, 0], \mathbb{R}^{n}\right)$ ) the origin of (4) is globally uniformly asymptotic stability for large $\Omega$, by making use of the following scaling property: if $x_{\Omega}\left(\phi, t_{0}\right)(t)$ is a solution of (4), then $k x_{\Omega}\left(\phi, t_{0}\right)(t)$ is a solution of (4), for any $k \in \mathbb{R}$. Notice that the space $\mathcal{C}_{r}\left(\left[-\tau_{0}-\right.\right.$ $\left.\delta, 0], \mathbb{R}^{n}\right)$ is invariant w.r.t. a multiplication of elements with a scalar.

\footnotetext{
${ }^{2}$ The only difference is that initial conditions are now taken in a function space. This does however not change the proof substantially. See [20], where such a generalization is made.
}

Finally, global asymptotic stability carries over to the space $\mathcal{C}\left(\left[-\tau_{0}-\delta, 0\right], \mathbb{R}^{n}\right)$, because of the following property: if $\phi \in$ $\mathcal{C}\left(\left[-\tau_{0}-\delta, 0\right], \mathbb{R}^{n}\right)$ then

$$
\begin{aligned}
\theta & \in\left[-\tau_{0}-\delta, 0\right] \rightarrow x_{\Omega}\left(\phi, t_{0}\right)\left(t_{0}+\tau_{0}+\delta+\theta\right) \\
& \in \mathcal{C}_{r}\left(\left[-\tau_{0}-\delta, 0\right], \mathbb{R}^{n}\right)
\end{aligned}
$$

and

$$
\max _{\theta \in\left[t_{0}, t_{0}+\tau_{0}+\delta\right]}\left|x_{\Omega}\left(\phi, t_{0}\right)(\theta)\right| \leq(|A|+|B|)\left(\tau_{0}+\delta\right)|\phi|_{s} .
$$

This completes the proof.

\section{REFERENCES}

[1] D. Breda, S. Maset, and R. Vermiglio, "Computing the characteristic roots for delay differential equations," IMA J. Numer. Anal., vol. 24, pp. $1-19,2004$.

[2] K. L. Cooke and Z. Grossman, "Discrete delay, distributed delay, and stability switches,” J. Math. Anal. Appl., vol. 86, pp. 592-627, 1982.

[3] K. Engelborghs, T. Luzyanina, and G. Samaey, "DDE-BIFTOOL v. 2.00: A Matlab package for bifurcation analysis of delay differential equation,” Dept. Comp. Sci., K.U. Leuven, Leuven, Belgium, T.W. Rep. 330, 2001.

[4] A. Goubet-Bartholoméüs, M. Dambrine, and J.-P. Richard, "Stability of perturbed systems with time-varying delays," Syst. Control Lett., vol. 31, pp. 155-163, 1997.

[5] K. Gu, V. L. Kharitonov, and J. Chen, Stability of Time-Delay Systems. Boston, MA: Birkhäuser, 2003.

[6] J. K. Hale and S. M. V. Verduyn, "Introduction to functional differential equations," in Applied Math. Sciences. New York: Springer-Verlag, 1993, vol. 99.

[7] P. Halmos, Measure Theory, ser. The University Series in Higher Mathematics. Princeton, NJ: Van Nostrand, 1966.

[8] S. Jayaram, S. G. Kapoor, and R. E. DeVor, "Analytical stability analysis of variable spindle speed machines," J. Manufact. Eng., vol. 122, pp. 391-397, 2000.

[9] V. L. Kharitonov and S.-I. Niculescu, "On the stability of linear systems with uncertain delay," IEEE Trans. Autom. Control, vol. 48, no. 1, pp. 127-133, Jan. 2003.

[10] V. B. Kolmanovskii and V. R. Nosov, Stability of Functional Differential Equations, ser. Mathematics in Science and Engineering. New York: Academic, 1986, vol. 180

[11] B. Lehman, J. Bentsman, S. V. Lunel, and E. I. Verriest, "Vibrational control of nonlinear time lag systems with bounded delay: Averaging theory, stabilizability, and transient behavior," IEEE Trans. Autom. Control, vol. 39, no. 5, pp. 898-912, May 1994.

[12] J. Louisell, "New examples of quenching in delay differential equations having time-varying delay," in Proc. 5th Eur. Control Conf., Karlshruhe, Germany, 1999, F1023-1.

[13] T. Luzyanina, K. Engelborghs, and D. Roose, "Computing stability of differential equations with bounded distributed delays," Numer. Algorith., vol. 34, no. 1, pp. 41-66, 2003.

[14] T. Luzyanina and D. Roose, "Equations with distributed delays: Bifurcation analysis using computational tools for discrete delay equations," Functional Differential Equations, vol. 11, no. 1-2, pp. 75-92, 2004.

[15] W. Michiels, K. Engelborghs, D. Roose, and D. Dochain, "Sensitivity to infinitesimal delays in neutral equations," in SIAM J. Control Optim., vol. 40, 2002, pp. 1134-1158.

[16] W. Michiels, S.-I. Niculescu, and L. Moreau, "Using delays and timevarying gains to improve the output feedback stabilizability of linear systems: A comparison," IMA J. Math. Control Inf., vol. 21, pp. 393-418, 2004.

[17] L. Moreau and D. Aeyels, "Trajectory-based global and semi-global stability results," in Modern Applied Mathematics Techniques in Curcuits, Systems, and Control, N. E. Mastorakis, Ed. Singapore: World Scientific, 1999, pp. 71-76.

[18] L. Moreau and D. Aeyels, "Practical stability and stabilization," IEEE Trans. Autom. Control, vol. 45, no. 8, pp. 1554-1558, Aug. 2000.

[19] L. Moreau and D. Aeyels, "Trajectory-based local approximations of ordinary differential equations," in SIAM J. Control Optim., vol. 41, 2003, pp. 1922-1945. 
[20] L. Moreau, W. Michiels, D. Aeyels, and D. Roose, "Robustness of nonlinear delay equations w.r.t. bounded input perturbations: A trajectory based approach," Math. Control Signals Syst., vol. 15, no. 4, pp. 316-335, 2002.

[21] S.-I. Niculescu, Delay Effects on Stability. A Robust Control Approach. Heidelberg, Germany: Springer-Verlag, 2001, vol. 269.

[22] S.-I. Niculescu, K. Gu, and C. T. Abdallah, "Some remarks on the delay stabilizing effect in SISO systems," in Proc. 2003 Amer. Control Conf., Denver, CO, pp. 2670-2675.

[23] S.-I. Niculescu, C. E. de Souza, J.-M. Dion, and L. Dugard, "Robust exponential stability of uncertain systems with time-varying delays," IEEE Trans. Autom. Control, vol. 43, no. 5, pp. 743-748, May 1998.

[24] W. Rudin, Real and Complex Analysis. New York: McGraw-Hill, 1966.

[25] J. Sexton and B. Stone, "The stability of machining with continuously varying spindle speed," in Ann. CIRP, vol. 27, 1978, pp. 321-326. 